\title{
Operational safety of high-rise buildings
}

\author{
Evgeniy Degaev ${ }^{1, *}$ \\ ${ }^{1}$ Moscow State University of Civil Engineering, Yaroslavskoe shosse 26, Moscow, 129337, Russia
}

\begin{abstract}
Risk of fire and smoke dangers are greater in high-rise rather than low-rise buildings. Use of high expansion foam delivered through elevator shafts can prevent or reduce smoke and fire damage and injury in high-rise buildings. Injection of foam with high multiplicity due to structural rigidity can accumulate between floors of buildings to prevent the spread of flame and smoke. Reaching an area of high-intensity flame, this foam is destroyed, resulting in the absorption of energy from the upward flow of products of combustion.
\end{abstract}

\section{Introduction}

\subsection{A high- Risk and danger of fire and smoke in high-rise buildings}

High-rise buildings are exposed to many dangers, but one of the highest dangers possible is fire. Existing statistics about fire emergencies in high-rise buildings confirm this level and kind of danger [1-5].

Fires in high-rise buildings especially are dangerous because, unlike in low-rise buildings, evacuation is quite difficult and fire control is complex. Fires in high-rise buildings feature rapid vertical development and require elaborate evacuation and rescue schemes. Tragic consequences can result from fires in high-rise buildings when fire and the products of combustion block human escape routes.

Extinguishing fires in high-rise buildings by traditional methods requires use of highpressure water, often transported and delivered over distances. High water consumption during fire extinguishing on the upper floors of high-rise buildings leads to flooding of the premises located below.

Elevator shafts in high-rise buildings play an important role in the transfer of air from lower to upper floors. Shafts become a bypass channel through which, even with the complete isolation of the stairwell zones, air flows from one zone to another to rapidly spread flame and smoke between floors [6-9]. Combustion products fill emergency exits, elevator shafts, and stairwells. Smoke and poisonous gases are transported vertically at speeds of several tens of meters per minute. Within a short time, a building can become filled with dense, noxious smoke. Recovering occupants of rooms is nearly impossible without respiratory protection for rescuers. The most intense smoke often rises to upper floors of high-rise buildings, where opportunities are limited for fire exploration, victim rescue, and access to necessary supplies of extinguishing media. Compounding these

* Corresponding author: degaev@inbox.ru 
problems are elevator equipment and fire protection systems that often fail during active fires.

\subsection{Some existing methods for mitigating risk and danger}

Stairwells and, especially, exits designs for high-rise buildings must accommodate the rapid removal of smoke and prevent the occurrence of other critical situations created by fire. The presence of two fire escapes is mandatory for high-rise buildings [10-12]. Skyscrapers must have available an adequate stock of personal protective equipment. Also, prior planning must confirm through engineering calculations that sufficient equipment available to combat fires.

Special requirements apply to materials employed to construct high-rise buildings, as well as to the materials from which the furniture and interior are made. For example, noncombustible materials are requirements for wall decorations, ceilings and floors in escape routes, and in elevator halls, lobbies, and technical floors. Nonflammable furniture and interior materials are necessary.

\subsection{Potential for use of high expansion foam}

High expansion foam offers one way to reduce damage from flame and smoke in high-rise buildings. Foam of high multiplicity traditionally is used to extinguish fires in confined spaces due to its high viscosity and capacity to limit the spread of fire inside a closed volume. Modern designs of generators of high expansion foam are capable of spreading a foam through smoke- and fire- polluted air. The advantage of these generators is that they require a low-pressure water line.

Regardless of the type of high multiplicity foam generator used, foam is not fully formed during the first seconds of operation in a smoky room. The better the design of the generator and the composition of the foaming agent, the shorter the induction period during which the presence of smoke prevents the formation of foam. During the induction period, smoke-filled air passes through the foam generator and, as a result of the action of the sprayed water jet, partial coagulation of smoke particles occurs. These partially coagulated particles settle on the floor of the room, together with water. As a result, the concentration of smoke in the room decreases.

Extinguishing a fire in a smoky room with a foam of high multiplicity occurs in two stages. The first foam is not fully formed and combustion products accumulated in the room as a result of the initial stage of combustion are pulled through the foam generator until a general outbreak of fire. After reducing the concentration of smoke in the air to the maximum permissible, the process of foam formation begins, but due to the preliminary impact of smoke on the material of the generator, foam formation occurs with less intensity than in the fresh air.

According to the experience of foreign manufacturers, the period of induction, a period that fills the room with foam at half of the room height, should not exceed 30 seconds. The amount of foam to be generated is determined from two calculations:

- The ability in 30 seconds to fill one-half of the volume of air in the room and the elevator shaft;

- The capacity to ensure the process of extinguishing the flame in the room and the elevator shaft does not exceed 60 seconds [13-17]. 


\section{Materials and Methods}

To give the foam a better spreadability, use of special foam generators is proposed, the design of which produces a foam of polydisperse structure with a viscosity that is much lower than a monodisperse structure [1].

Generator polydisperse vysokokratnoy vortex type foam consists of a housing 1 (Fig. 1.), Constructed as a prismatic shell (e.g. a rectangular prism, in which the cross section square). On the one hand to the housing 1 is coupled foam solution dispenser which is configured as a vertically disposed, at least one inlet duct 3 with the support 2 and the grid flanges (not shown) for connection to the system of extinguishing liquid foaming solution. Perpendicular to the input conduit 3 horizontally arranged rigidly associated with it, at least two additional line 5 with plugs at both ends, wherein the distributor foam solution fits the inner contour of the housing 1 and the inner diameter of the cavity inlet 3 and 5 additional conduits are equal, and cavities themselves interconnected. Input to the inner cavity 3 and 5 additional piping connected from the sleeve casing 1 and parallel to its axis of bends: shortened 4 , medium 6 , and 7 long, 8 ending with nozzles oriented toward the guide unit 10 , the mesh.

The guide device 10 consists of three concentric, coaxially arranged in a pyramidal shells generating grids: outer 15, inner 11 and intermediate 14. Each of the shells comprises a wire skeleton of the two bases in the form of a square and the side edges of a truncated pyramid surface. The outer shroud 15 , a large base attached to the body 1 , and its minimal second base 9 is simultaneously large intermediate base 11 with the side edges of mantle 12 and its smaller base is both large base inner shell 14 with a lower base 13. Generating mesh in the form of truncated pyramidal surfaces are arranged so that their vertices lie on an axis coinciding with the axis of the housing, the vertex direction of the outer 15 and inner 14 coincides generating grids, and the intermediate vertex 11 mesh hydrochloric generating oppositely directed vertices outer 15 and inner 14 generating meshes with foam solution towards the distributor. Fastening body 1 to distributor foam solution is carried out by means of fasteners 16 .

Each of the nozzles 8 consists of a casing 17 and coaxially arranged and rigidly associated at the top with an inlet fitting 18, a cylindrical bore 19 connected to diffuser 20 formed axially in the housing 17 . The lower housing portion 17 disposed axisymmetrically nozzle body 21 . The nozzle 21 is formed with two-stage, and insert-coaxial swirler 22, a diffuser, wherein the first stage diffuser 26 is an extension of the expanding tapered bore 23 formed within the insert-swirler 22, and the second stage 27 it is a continuation of the diffuser of the first stage 26, the helical thread 28 formed on its inner conical surface.

Box-swirler 22 is arranged in a central cylindrical bore 24 of the housing 17 and has a threaded outer peripheral helical channels 25 , and within the insert-swirler 22 formed coaxially expanding tapered bore 23 for supplying liquid from the cylindrical bore 19 formed in the fitting 18. The insert-swirler 22 fixed in the lower housing portion 17 by a nozzle 21. Box-swirler 22 is made of wear-resistant material. 

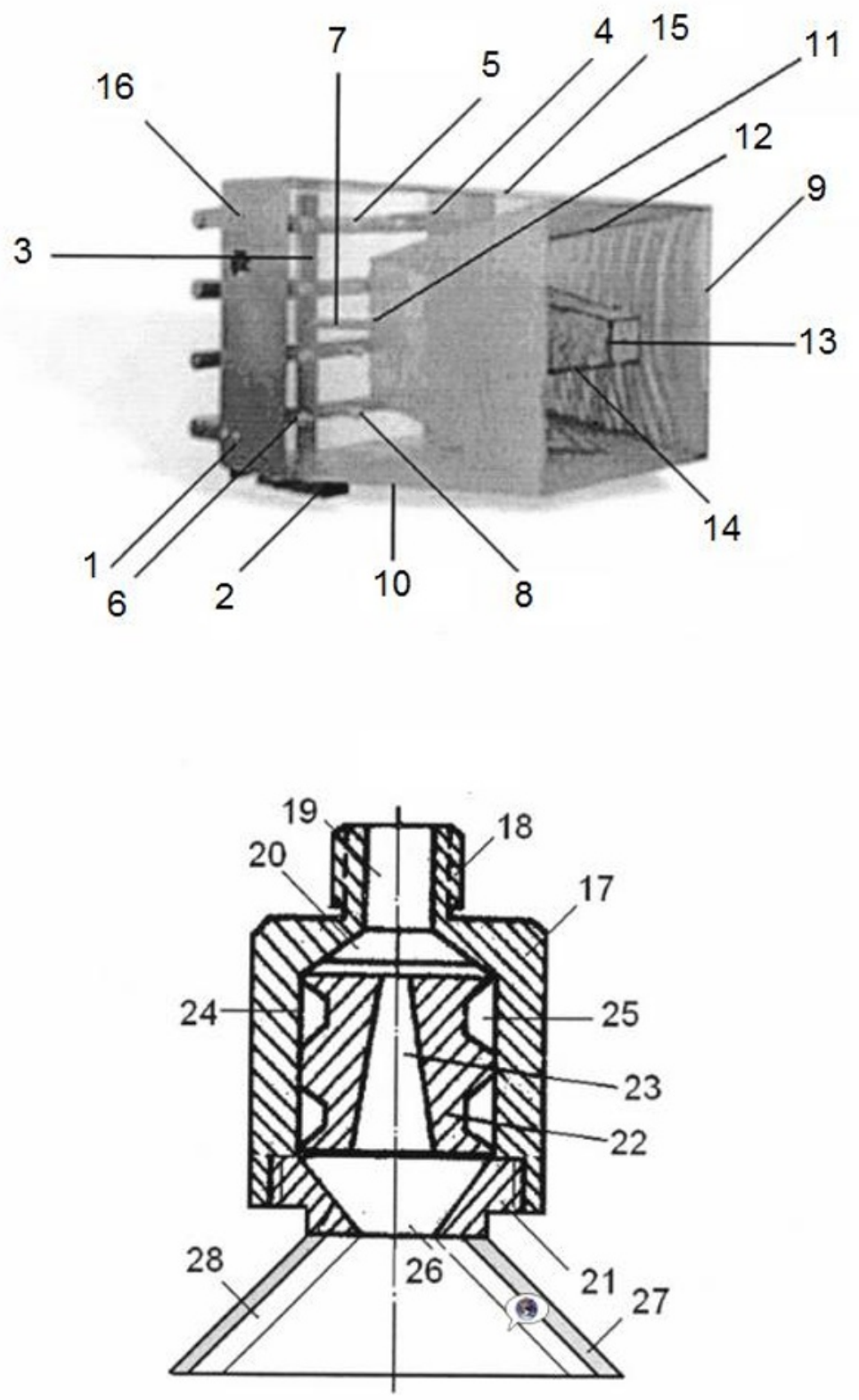

Fig. 1. Polydisperse foam generator.

This design obtains a foam with a variable structure, which alternates areas with small bubbles and the inclusion of large formations. This structure dramatically reduces the viscosity and ultimate shear stress of the foam, allowing the foam to spread quickly over the burning surface and form barriers to the spread of flame in the elevator shafts $[3,4]$.

The device consists of a metal housing and a thin, perforated holes in metal sheet or metal mesh located behind the generator housing and completely covering the entire section of the metal housing. The perforated sheet metal is hermetically connected to the generator housing, so that all the solution and air pass through the holes in the perforated metal sheet. 
The foam generator works due to the pressure of a sprayed jet of aqueous solution of the foaming agent, which is formed by means of special nozzles. The air is drawn (ejected) into the generator housing by a spray jet of water. As a result of the passage of the solution and air through the perforated metal surface foam is formed. The average diameter of the foam bubbles is two to three times larger than the diameter of the holes in the metal.

Fires in high-rise buildings spread mainly from the outside of the building, with the flow of smoke moving up to the top of the building. Sections of multi-story buildings are separated by elevator shafts, which also support the flow of smoke and flame to create an upward column of flame [6-8].

Depending on the height of the building, it is advisable to install high multiplicity foam generators every five floors. Some generators will fill the space below the elevator with foam, and other generators will fill the opening above the elevator [11-13].

\section{Results and discussion}

Given that the height of the foam column can reach several meters, it is necessary to select foaming agents that are able to form a foam with a high capillary pressure limit. An increase in the height of the foam layer leads to a synchronous increase in hydrostatic and capillary pressure in the foam channels and the rupture of foam films.

The higher the height of the foam layer, the higher the rate of destruction of the foam. Assuming that the specific rate of hydrostatic destruction of the foam, Uh, is proportional to the height, h:

$$
\mathrm{Uh}=\beta \mathrm{hh},
$$

we write the equation of the material balance of the foam, taking into account the fact that a certain part of the foam is destroyed in the surface layer:

$$
\mathrm{q} d \tau=\text { So } d h+\text { SohUh } d \tau,
$$

considering that $\mathrm{Uh}=\beta \mathrm{hh}, \mathrm{a} \mathrm{q} / \mathrm{S}=\mathrm{J}$, we get:

$$
J \mathrm{dt}=\mathrm{dh}+\beta \mathrm{hh} 2 \mathrm{~d} \tau
$$

where $\mathrm{S}$ is the area; $\mathrm{J}$-intensity.

Integrating the differential equation arising from equation (3) in the range from $\tau=0$ to $\tau=\tau$ Т and from $\mathrm{h}=0$ до $\mathrm{h}=\mathrm{H}$, we obtain:

$$
\tau \mathrm{T}=\frac{1}{\beta_{h}} \frac{1}{2 \sqrt{J / \beta}} \ln \frac{\sqrt{J / \beta}+H}{\sqrt{J / \beta}-H},
$$

where $\mathrm{H}$ is the height of the room.

Time $\tau \rightarrow \infty$ when $\sqrt{J / \beta}=H$, so the critical intensity of the Jcr is determined from the formula:

$$
\mathrm{J}=\mathrm{H} 2 / \beta \equiv \mathrm{Jcr} \text {. }
$$

The value of the coefficient $\beta \mathrm{h}$ is determined by the limit value of the capillary pressure, which can withstand foam [1]. 


\section{Conclusions}

Fires of high-rise buildings are dangerous, and extinguishing these fires is a complex process. The use of traditional means and methods of extinguishing is often not sufficiently effective and efficient [7-9]. Taking into account that the facade of a building is mostly filled and because elevator shafts remain without due attention, the spread of flame and smoke on floors in a high-rise building is created and maintained in elevator shafts.

According to our calculations, the use of modern generators of high-quality foam, installed every five floors, can provide rapid filling of elevator shafts, eliminating the possibility of smoke and flame inside the building and promoting the safe evacuation of people and the preservation of the bearing capacity of the building [12-16].

\section{References}

1. A.F. Sharovarnikov, V.P. Molchanov, S.S. Voevoda, S.A. Sharovarnikov, Extinguishing fires of oil and oil products. Izd. house "Kalan", p. 448 (2002)

2. M.A. Anisimov, E.N. Degaev, Warehouse Premises and Tank Farms Fire Safety Problem. Vestnik MGSU, 13, № 10 (121), pp. 1243-1250 (2018) DOI:10.22227/19970935.2018.10.1243-1250

3. V.G. Borkovskaya, E.N. Degaev, I. Burkova, Environmental economic model of risk management and costs in the framework of the quality management system. MATEC Web of Conf., 193, 05027 (2018) DOI: https://doi.org/10.1051/matecconf/201819305027

4. V. Polyakova, E.N., Degaev, E.H. Pierre, Reduction of Ecological and Economic Risks in Utilization of Solid Domestic Wastes and Construction Waste. MATEC Web of Conf., 251, 06017 (2018) DOI: https://doi.org/10.1051/matecconf/201825106017

5. E.N. Degaev, New classification of Foaming Agents for Fire Extinguishing. MATEC Web of Conf., 193, 02032 (2018) DOI: https://doi.org/10.1051/matecconf/201819302032

6. E.N. Degaev, A. Orlov, E.H. Pierre, A. Pleshivtsev, Ecological and Economic Risks of Fire Protection of Warehouses and Tank Parks. MATEC Web of Conf., 251, 06013 (2018) DOI: https://doi.org/10.1051/matecconf/201825106013

7. E.N. Degaev, A. Suvorova, A. Suhova, Influence of total head of foam on optimum intensity and minimum particular expense solution size of foamer. IOP Conf. Ser.: Mater. Sci. Eng., 365032019 (2018) DOI: https://doi.org/10.1088/1757899X/365/3/032019

8. E.N. Degaev, V. Razvalyaeva, S. Sabenina, Formation of water film from aqueous film forming foam drops on the surface of oil products. IOP Conf. Ser.: Mater. Sci. Eng., 365062037 (2018) DOI: https://doi.org/10.1088/1757-899X/365/6/062037

9. A. Kattge, E.J.Degajev, Der einfluss der höhe der schaumabgabe auf die optimale intensität und den minimalen verbrauch von schaumlöschmitteln. Zeitschrift für Forschung, Tech. und Manag. Brandschutz, № 1, pp.150-152 (2016)

10. V.G. Borkovskaya, Complex models of active control systems at the modern developing enterprises. Ad. Mat. Res., 945-949, pp. 3012-3015 (2014) DOI: 10.4028/www.scientific.net/AMR.945-949.3012.

11. V.G. Borkovskaya, Environmental and economic model life cycle of buildings based on the concept of "Green Building". Appl. Mech. and Mat., 467, pp. 287-290 (2014) DOI: $10.4028 /$ www.scientific.net/AMM.467.287 
12. V.G. Borkovskaya, W. Roe R. Bardenwerper, Interactive Teaching of Risk Management in the Russian Construction Industry. IOP Conf. Series: Materials Scie. and Eng., 365, 062030 (2018) doi:10.1088/1757-899X/365/6/062030

13. E.A. Korol, A.A. Pleshivcev, Multiple-criteria decision analysis of the transformable low-rise building technological construction process. MATEC Web of Conf., 193, 03042 (2018).DOI: https://doi.org/10.1051/matecconf/ 201819303042

14. E.A. Korol, N. Shushunova, Green Roofs: Standardization and Quality Control of Processes in Green Construction. MATEC Web of Conf., 106, 06014 (2017) DOI: https://doi.org/10.1051/matecconf/201710606014

15. E. Korol, D. Mostovoy, A.A. Pleshivcev, Technological parameter optimization of multilayer enclosure structures with the multiple-criteria decision analysis. MATEC Web of Conf. 170, 03031 (2018) DOI: https://doi.org/10.1051/matecconf/201817003031

16. Patent RU141353U1. Generator polydisperse vysokokratnoy vortex type foam. 